\title{
PSYCHOLOGICAL MECHANISM OF THE MEDIA INFLUENCE
}

Jose Mathews*
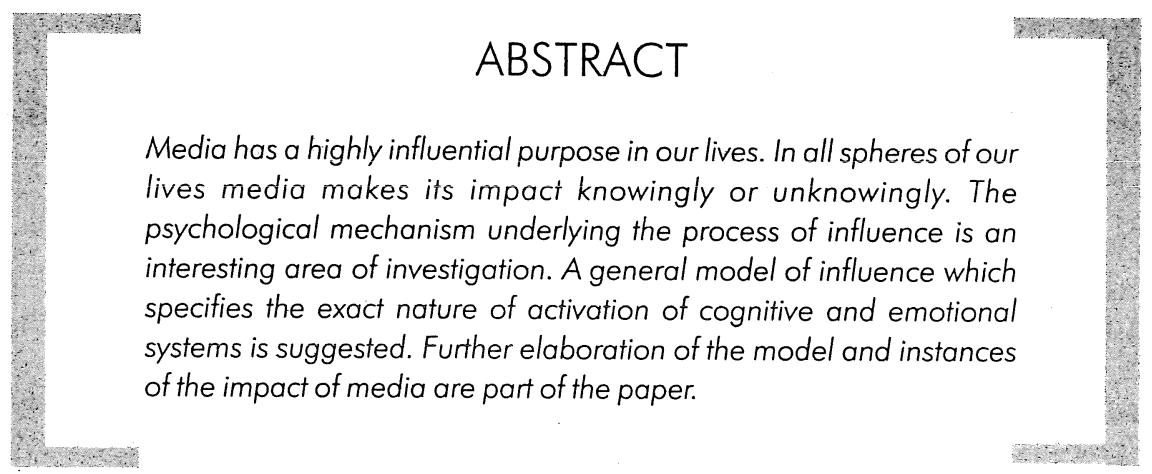

\section{Introduction}

In the print and audio visual media we come across a series of programmes that have a direct bearing on our daily lives. It is an incontrovertible fact that our lives are shaped, modified and developed by the media: Media is the most important

* Lecturer, Dept. of Psychology, Christ College, Bangalore-29. 
source of information. For anything and everything we have to turn to the media. The media is always at our disposal.

What is startling about the media is that it influences us in many ways that are unimaginable and uncontrollable. The barriers of our minds are broken by the onslaught of the media and we are in a helpless situation as to what is good and what is evil. The mechanism of the media projection fails us to cope with the barrage of hyper flow of information.

The influence of the media can be in the direction of productivity and unproductivity. Media inculcates healthy practices. A major section of our viewers are victims of this fanciful show on the earth.

It is proposed to show how the media influences us. What are the psychological bases or processes of channels of influence. It is an interesting aspect because media has differential effects. Who are the individuals easily affected or programmed by the media? What is the basic mechanism of influence? These are the questions answered here.

\section{Different Forms of Media}

We can recognise the existence of different forms of media in our environment. Different technological arrangements and types of media communication have been categorised (Surmanek, 1996). Besides there are multiple channels in the air.

\section{Network TV}

A network is any group of local television stations electronically joined to broadcast the same programs usually simultaneously.

\section{Network Cable TV}

Cable networks also transmit programming and commercials to local market via satellite. The local cable operator receives the signal via a satellite dish and retransmits it to those households that subscribe to the cable service.

\section{Regional Cable TV Networks}

In concept and transmission technique regional cable TV networks are identical to national cable TV networks, but it is beamed to selected geographic areas. 


\section{Multiple Channels}

The viewer has the facility to select any channel from the plethora of channels offered by different TV networks in news, entertainments, education, geography, sports etc.

\section{Radio Broadcast}

The radio frequencies have the largest coverage in any country. In India it has the highest reach.

\section{Magazines and Periodicals}

The colourful, picturesque and informative magazines being circulated deal with different aspects of life. These publications have a tremendous impact on the minds of the readers. The subjects included in these are quite innumerable.

\section{News Papers}

Besides the commonly known daily newspapers with editions in the morning and evening, there are weekend newspapers.

\section{Billboards and Posters}

Billboards and posters displayed in public places in painted and illuminated fashion catch the attention of many.

\section{Interactive Media}

Technological advances in video, audio, and data transmission techniques are causing major changes in how consumers will receive media, what consumers will receive and how consumers will be able to respond to messages. People are travelling on an electronic information super highway where there is an integration of T.V., radio, computers, telephones, fax machines and much more. Combining the innovations with a TV set and a computer, the average home not only will be able to receive transmissions but also will be able to react to transmission. 


\section{The Basic Chracteristics of Persuasion}

The process of influence has assumed the nature of a tricky art as well as a highly sophisticated activity, enmeshed in a series of subtle, sublimindal and explicit ways. No one can overlook the basic fact of influence because it is a day-to-day reality.

Certain characteristics have been delineaed by psychologists to bring out the scientific aspect of persuasion. A person with expertise and good credentials can influence your behaviour. Popular personalities who are able to hold the attention of others are persuasive. Attractive and charismatic persons tend to change the opinion of others. If the persuasive intent is not so obvious and when the viewers do not 'see' the object of influence, it gradually results in influence.

Bidirectional issues or statements which project the different aspects have more weight than statements which bring out only one aspect.

Fear arousing stories, serials and episodes have more captivating power. In the stirred state, there is no active utilisation of the cognitive resources. We are carried away by emotions. Many persons cry at the end of a tragic love story.

Personality characteristics of the viewers and listeners play an important part in influence. Individuals with low self-esteem, high suggestibility tendency, low intelligence and low self concept tend to be disturbed by the media gimmicks.

\section{Cognitive response analysis}

An approach that determines the mental processess in persuasion is called the cognitive response analysis (Baron and Byrne 1988). The volume of thinking involved in a particular programme, both during the act and after the act, determines its influence upon you. There is no passive absorption of message. We twist, change, assimilate and accommodate the information given to us. The deliberation and reflection carried out decides the way a serial or a talk influences us.

According to the elaboration likelihood model of the cognitive response analysis, there are two different "routes" to persuasion. The central route involves careful and thoughtful consideration of the issue. When the advertisement is at the centre of the person's attention, it takes a central route. In instances of low consideration and no careful thinking, the route taken is called a peripheral route. 


\section{Distraction theory of influence}

Media can change our behaviour by a process called distraction activity. It does not demand our considered judgements or active interest. By a process of confusion and rapid changes in attention one can be easily enamoured of the message the person is trying to impress upon us. This sudden change in our decision occurs because of the distraction, the person is trying to cope with. We are moved to restore balance by aligning with a particular action. This process of influence can happen along predicted lines and in unpredictable ways.

\section{General Model of Influence}

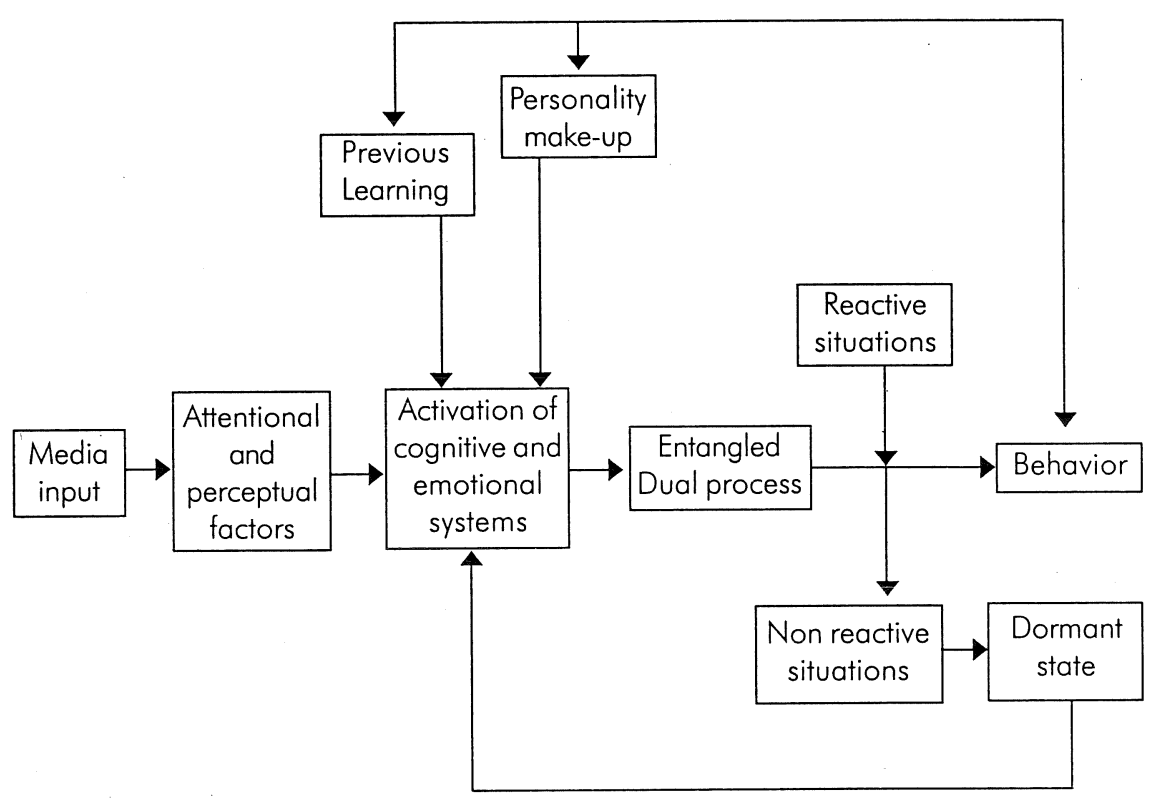

Fig. 1 General Model of Influence 


\section{Explanation}

\section{Media Input}

It consists of being exposed to the visual and auditory media. It specifically refers to programmes telecast on television and broadcast on radio. The print media provides the third source of input to a person.

\section{Attentional and perceptual factors}

Attention involves a process of selection. The attenuation model supposes that non selected channels are not completely shut off but are simply turned down or dampened. The filter in the filter theory is an all-or-none affair. What is not selected is turned out completely. (Best, 1992)

At the physiological level, attention is a process of being channeled to a single sensation that activates any of the sensory modality. From a multitude of sensory channels except that which has a prepondering effect, all other sensations impinged on the senses are eliminated or fall below the threshold. They exist at the periphery of the field of attention. Those sensations centring on the field of attention are processed.

If we take the television screen some pictures or conversations are centred in the field of attention. Our attentional system has only limited resources to focus on the inputs. Similar to our way of changing the channels and fixing on a specific one, the mechanics of attention work in such a way that, we select a specific input for processing.

Attention being a self-selected activity, one has to recognise the internal factors responsible for selecting a particular stimulus and how do we maintain our attention over a period of time. The need pattern of the person influences the selection of a news item or a programme. Closely related to the need pattern, it is the motivational dynamics of a person that determines the attentional processes. Achievement oriented need leads one to view programmes related to knowledge development.

Personality of the person plays an important role in directing our attention. Individuals with a sense of humour are able to watch events related to laughter.

Other internal factors that influence our attention are the mental set of the person, intelligence, interest and our general disposition. 
There are aspects external to us that control the attentional mechanism. Movement easily gets noticed and when all the individuals are in action, that which is novel, familiar or unfamiliar are attended to. Size and intensity of the stimuli determine our attention.

In interpreting and giving meaning to a stimuli, there is an innate mechanism to organise the perceptual field. Besides the context influences our perception. The specific state of the emotional experience colour our perception. Previous experiences help us to attribute meaning to something.

\section{Activation of cognitive systems}

Cognition refers to the process by which the sensory input is transformed, reduced, elaborated, stored, recovered and used. The transformation of the physical stimulation produces more than just a neural code. It also results in the creation of a cognitive code. Once created, the cognitive code can be reduced or elaborated. Elaboration of a cognitive code refers to the relating of specific mental events. In many cases, cognitive codes can be stored, recovered and reconstructed (Best, 1992).

Schema theory helps to know the structure and processes of the cognitive systems. A schema is an organised collection of one's beliefs and feelings about something. New cognitive structures, that is schemata, build on the structures acquired earlier.

Self schema is a powerful factor in the way people understand their social world. The other schemata operative are person schema, role scheme, and event schema (Baron and Byrne, 1988).

Activation of a schema in a given situation give rise to a person's expectations, influences and actions in that situation. Such schemas exist at many levels, and schemas at different levels can simultaneously be active in influencing us (Friedman and Schustack 2003).

Serials, features and shows that have some relation to the self attract more attention, produce more thought and are remembered better than other events. This is an occasion for self-schema activation. Upon reception of the input, the organised system and beliefs and thoughts undergo some kind of a change leading to an unlocking activity. The stored system of thoughts is brought to the open. The person continues to be under its influence.

It is not the activation mechanism alone that is of interest to us. New schemas are formed following the learning activity. The schema of family life or interpersonal 
relations are being changed consequent to the active intertest we show in TV. productions. Whether for good or bad we are being influenced by the media in many ways. If we make a comparison between the young and the old, we see entirely different systems active, one due to the influence of the media, the other based on our traditions.

\section{Emotional system}

An emotion is a stirred up state. The first component of emotion is the bodily arousal and the second aspect is the psychological experience of pleasantness or unpleasantness.

An influential theory of emotion was proposed by Schachter and Singer. The arousal part is largely the same for all emotions. The cognitive label comes from interpreting the situation. The bodily arousal is the on-off control which decides whether there will be any emotion or not. The cognitive part is like the channel selector, deciding which of the various possible emotions is taking place.

In cognitive theory, the importance of cognitive interpretation of circumstances in determining arousal levels is central (Malim and Birch, 1992).

Attribution theory suggests that we attribute causes to all events that happen, whether or not we have adequate information to do so. Weiner sees emotion as coming from these attributions. Attributional processes stemming from the motivational dynamics decides the emotional experience.

\section{Previous learning}

Learning results in stable, consistent pattern of behaviour. Learning can be maladaptive or adaptive, contingent on reinforcement or inner resources and it can be conscious or unconscious. Each person has separate learning experiences.

\section{Personality make-up}

The consistent and varying modes of adjustment with the environment and the nature and dynamism of the psychophysical systems constitute the personality makeup of a person. The inner ease and the conflict within the person and the graceful and disgraceful behaviour can be differentially reactive to the media.

Previous learning experiences and personality make-up moderate the activation of cognitive and emotional systems. 


\section{Entangled dual process}

In the activated system, cognition and emotion get entangled and give rise to a dualistic process where there is a process of relational influence.

People who feel good find it especially easy to remember good things about themselves and others. They tend to judge things in a more positive light than people with bad feelings. A pleasant state results in a positive evaluation. An unpleasant state leads to a negative evaluation.

Bad feelings have complex effects. Negative emotions lead to conflicting tendencies. III tempered feelings have their source in hatred or aversion.

A contaminated cognitive system can be the cause of aggressive expressions just like a purified system can produce a state of peace.

An emotional state, negative or positive has its basis in cognition. And cognitive activity, whether resourceful or nonresourceful has its basis in the emotional experience.

\section{Reactive situation}

The overt expression of the entangled dual process takes place in situations that demands it. There has to be a precipitating factor. A child who is influenced by the Bharathanatyam performance of a danseur will enroll for a course when she sees a dance school.

\section{Non-reactive situation}

In the absebce of the proper stimulus situation, the person may not react.

\section{Dormant state}

The traces will be left behind if the activated state do not find any expression. It will remain with the person in a diffused, disused and dormant form.

\section{Enactment of the behavior}

The media input that makes an influence on the person will be expressed by the person in direct and indirect ways. The behaviour may be expressed in talking or other ways. 


\section{The State of Activation Model}

This model is an elaboration of the general model of influence. This derived mechanism deals with the specific nature of the activated cognitive emotional systems. It consists of simple thoughts and complex thoughts and basic emotions and complex emotions. The thoughts are explained in polar opposites to include both the media inputs socially good and socially bad. The same theme can produce different reactions is to be understood with reference to the earlier part of the model.

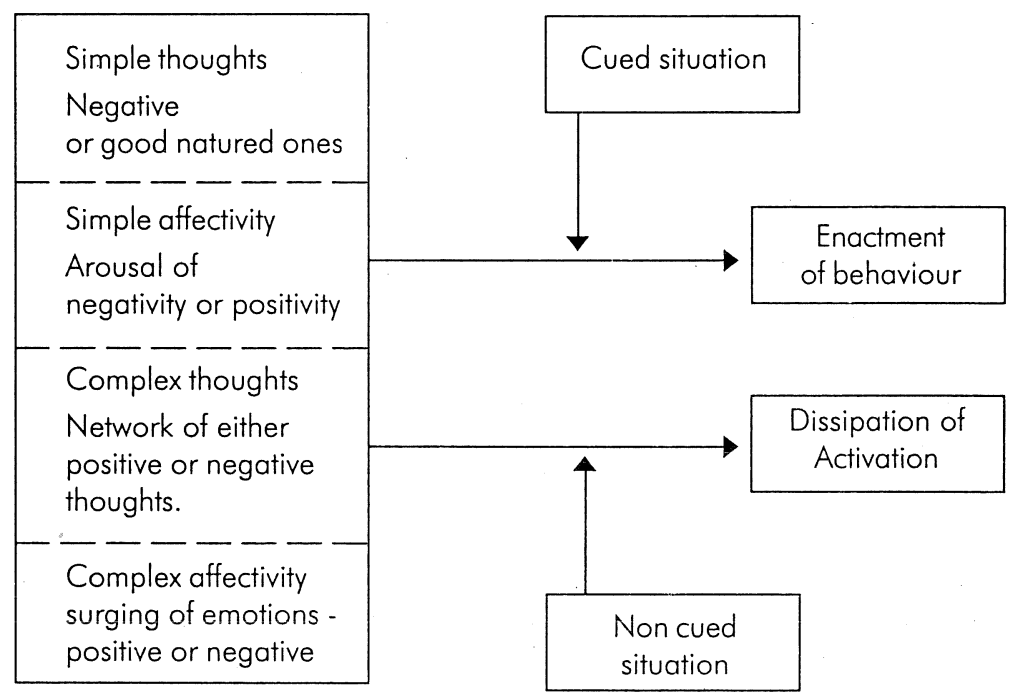

The State of Activation

The generation and maintenance of simple and complex thoughts is very much related to the schema or cognitive structures of the person. The thoughts have two qualities, either they are transient or they are long lasting. Te former pattern of thoughts does not have much impact. The latter may continue to influence the person. In the case of simple schema, we have simple thoughts and complex schemata can lead to complex or a network of thoughts. The complex nature of the affectivity is encircled with the mesh of thoughts. 


\section{Instances of media influence}

A growing body of explicit instances can be found in our lives to demonstrate the impact of media. Besides the indirect ways, the cases of direct influence are too many to be included here.

First of all, our life styles are formed and altered by the visuals and information provided in the media. The characteristic pattern of our lives can be traced to the media.

Secondly, our goals and careers can be induced by the media. The decision making activity is largely based on the information provided by the media.

Thirdly, our consumption pattern, use of household goods and the type of buildings that we build is related to what the media is providing us.

Fourthly, the media is influential in deciding our entertainment activities and the way we spend our time.

Fifthly, when media displaces our traditions, new forms of culture and customs are brought in by the media.

\section{Conclusion}

Media influences our lives. The mechanism explained to show how we are influenced by the media is not the complete answer. The model may be modified in the light of evidences and other ways.

\section{References}

1. Baron, R.A., Byrne, D (1988). Social Psychology, $5^{\text {th }}$ Ed., New Delhi: Prentice Hall of India.

2. Best, J.B. (1992). Cognitive Psychology, $3^{\text {rd }}$ Ed., NewYork: West Publishing Co.

3. Friedman, H.H.S. \& Schutack (2003). Personality, $2^{\text {nd }}$ Ed., Delhi: Pearson Education

4. Malim, T., \& Binch, A., (1998). Introductory Psychology. London: MacMillan Press.

5. Surmanek, J. (1996). Media Planning: A Practical Guide. NTC Business Books. 